\title{
Implementierungsstrategien für effektiven Datenschutz
}

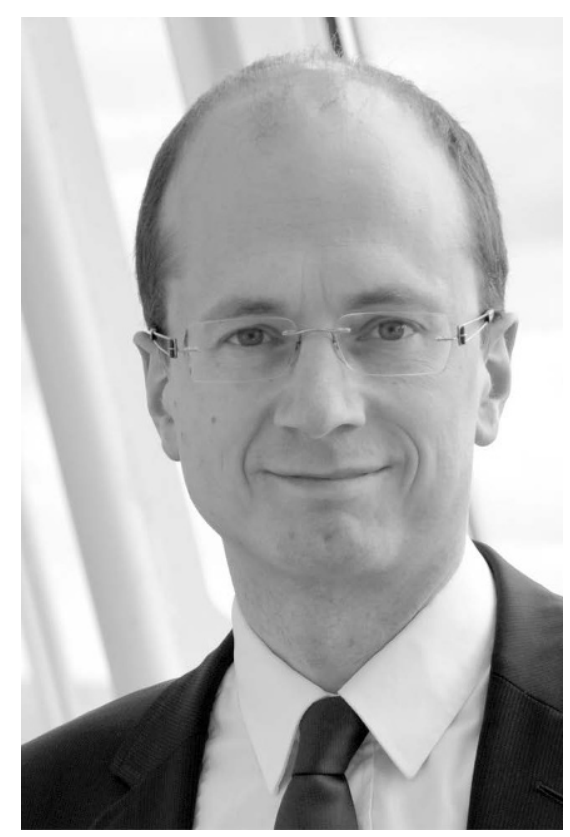

Eineinhalb Jahre nach ihrem Geltungsbeginn hat die Diskussion um die Datenschutz-Grundverordnung etliches von der anfangs teilweise zu beobachtenden Hysterie verloren. Zugleich hat sich die Verordnung allerdings fest als fortwährende Quelle offener Rechtsfragen etabliert. Dies gilt zum einen für die wenigen, überdies sehr allgemeinen und häufig abwägungsoffenen materiellrechtlichen Bestimmungen, zum anderen aber auch für die teilweise innovativen verfahrensrechtlichen Vorgaben.

Viele dieser Fragen werden in den nächsten Jahren nach und nach gerichtlich geklärt werden. Dieser Klärungsprozess wird aber schon mangels eines justiziellen Initiativrechts notwendigerweise erratisch bleiben. Dies stellt die Praxis des Datenschutzes vor erhebliche Herausforderungen: Wer zu lange auf gerichtliche Klärung wartet, setzt sich möglicherweise dem Vorwurf aus, in rechtswidriger Weise untätig geblieben zu sein. Wer umgekehrt zu früh technische und organisatorische Maßnahmen ergreift, läuft Gefahr, einen später gerichtlich festgestellten Maßnahmebedarf zu verfehlen, und dann entweder teuer nachsteuern zu müssen oder (aus Datenschutzsicht vorteilhaft, aber betriebswirtschaftlich problematisch) überobligatorisch tätig gewesen zu sein.

Der Hinweis, dass vergleichbare Probleme mit praktisch jeder grundlegenden Rechtsreform einhergehen, ist so zutreffend wie für den Praktiker nutzlos, den es bei der Lösung seiner eigenen Fragen nicht weiterbringt, dass sich vergleichbare Herausforderungen in anderen Rechtsgebieten stellen. Die Aufgabe dürfte darin liegen, zum einen verschiedene Implementierungsstrategien zu erarbeiten und zum anderen ein zumindest tastendes Gefühl dafür zu entwickeln, wann welche dieser Strategien im Einzelfall angebracht ist. Bestimmte Verarbeitungssituationen mögen stark technikgetriebene Lösungsansätze erfordern. In anderen Situationen können technische und/oder ökonomische Probleme einem effektiven technischen Datenschutz im Wege stehen, sodass nach rechtlich-organisatorischen Absicherungen gesucht werden muss. Manchen Verantwortlichen wird man für ihre Verarbeitungsprozesse ein vorläufiges "Weiter so" mit den unter der alten Rechtslage entwickelten Schutzinstrumenten empfehlen können, andere mussten und müssen völlig umdenken.

Verschiedene Implementierungsstrategien lassen sich derzeit bereits in der Praxis beobachten und sind als solche - gerade mit Blick auf die neuen Vorgaben des europäischen und nationalen Datenschutzrechts - naturgemäß auch Gegenstand wissenschaftlicher Analyse. Die Schwerpunktbeiträge dieses Hefts sind aus dem Track "Informatik mit Recht" der diesjährigen Jahrestagung der Gesellschaft für Informatik (Gl) „,50 Jahre Gesellschaft für Informatik - Informatik für Gesellschaft“ in Kassel hervorgegangen und befassen sich aus unterschiedlichen Blickwinkeln mit derartigen Herausforderungen.

Aus technischer Perspektive erörtert der Beitrag von Gerl und Meier (Universität Passau) Methoden zur Umsetzung der Informationspflichten der Datenschutz-Grundverordnung am Beispiel einer für Menschen und Maschinen gleichermaßen lesbaren Layered Privacy Language. Derartige Ansätze könnten eine Lösung für das Problem sein, dass die Menge der bereitgestellten Informationen sich ab einem gewissen Punkt umgekehrt proportional zur Menge der tatsächlich von Menschen wahrgenommenen Informationen verhält. Stach (Universität Stuttgart) stellt Konzepte vor, die den Datenschutz bei loT-Zeitreihendaten erhalten sollen, ohne die Servicequalität unnötig einzuschränken. Dies soll dem Problem vorbeugen, dass sich in langfristig erhobenen Daten in aller Regel hochspezifische, auf konkrete Personen und Ereignisse beziehbare Informationen wiederfinden. Der Text von Braun, Scepanko$v a$, Holl und Matthes (TU München) geht der Frage nach, ob Technologien des "LegalTech" ein Mittel des (Verbraucher-)Datenschutzes sein könnten, indem Vertrags- 
bedingungen automatisiert auf problematische und/oder rechtswidrige Klauseln untersucht werden. Dies könnte zu einer Erweiterung des Verständnisses von Datenschutz durch Technikgestaltung führen.

Ein bisher wenig beleuchtetes Thema behandelt der Beitrag von Borell (Datenschutzberatung Moers) und Schindler (Universität Kassel). Die Jl-Richtlinie hat die nationalen Gesetzgeber dazu gezwungen, etliche neue und detailliertere Pflichten für technische und organisatorische Maßnahmen für die polizeiliche Datenverarbeitung vorzusehen. Hier liegen erhebliche Chancen für die datenschutzkonforme Verarbeitung in den Behörden, es bedarf aber noch neuer Konzepte, um auch im Sicherheitsbereich Datenschutz durch Technikgestaltung einzuführen und wirksame Datenschutz-Folgenabschätzungen einzusetzen. Schließlich befassen sich Schomberg, Barev, Janson und Hupfeld (Universität Kassel) mit den Möglichkeiten und Grenzen eines Datenschutzes durch Nudging. Art. 25 Abs. 2 DSGVO enthält ja seit neuestem sogar eine bußgeldbewehrte Rechtspflicht, betroffene Personen durch datenschutzfreundliche Voreinstellungen zu nudgen. Ob und in welchen Szenarien ein solches "Schubsen“ möglich und legitim ist, bedarf aber weiterer Diskussionen.

Ergänzt werden die Schwerpunktbeiträge um Texte, die ebenfalls Implementierungsfragen in den Blick nehmen. Wessels (Universität Bremen) stellt erste Gerichtsentscheidungen zu Schmerzensgeldern nach Art. 82 Abs. 1 DSGVO vor. Auch wenn diese bisher zurückhaltend ausfallen, könnte hierin ein deutlicher Anreiz für die Beachtung des Datenschutzrechts liegen. Durmus (NOTOS Xperts), Selzer und Pordesch (Fraunhofer) behandeln schließlich die vielfach ungelösten Probleme der Anwendung datenschutzrechtlicher Löschvorgaben auf gespeicherte E-Mails und den Nachweis der entsprechenden Umsetzung.

Die allen Arbeiten implizit zugrundeliegende Frage ist, wie man mit den verschiedenen Strategien zu einem effektiven Datenschutz gelangen kann. Die Texte liefern damit Beiträge zum Werkzeugkasten der datenschutzrechtlichen Instrumente und regen - so ist zu hoffen - zum Nachdenken über die eigene Instrumentenwahl an.

\section{Gerrit Hornung}

